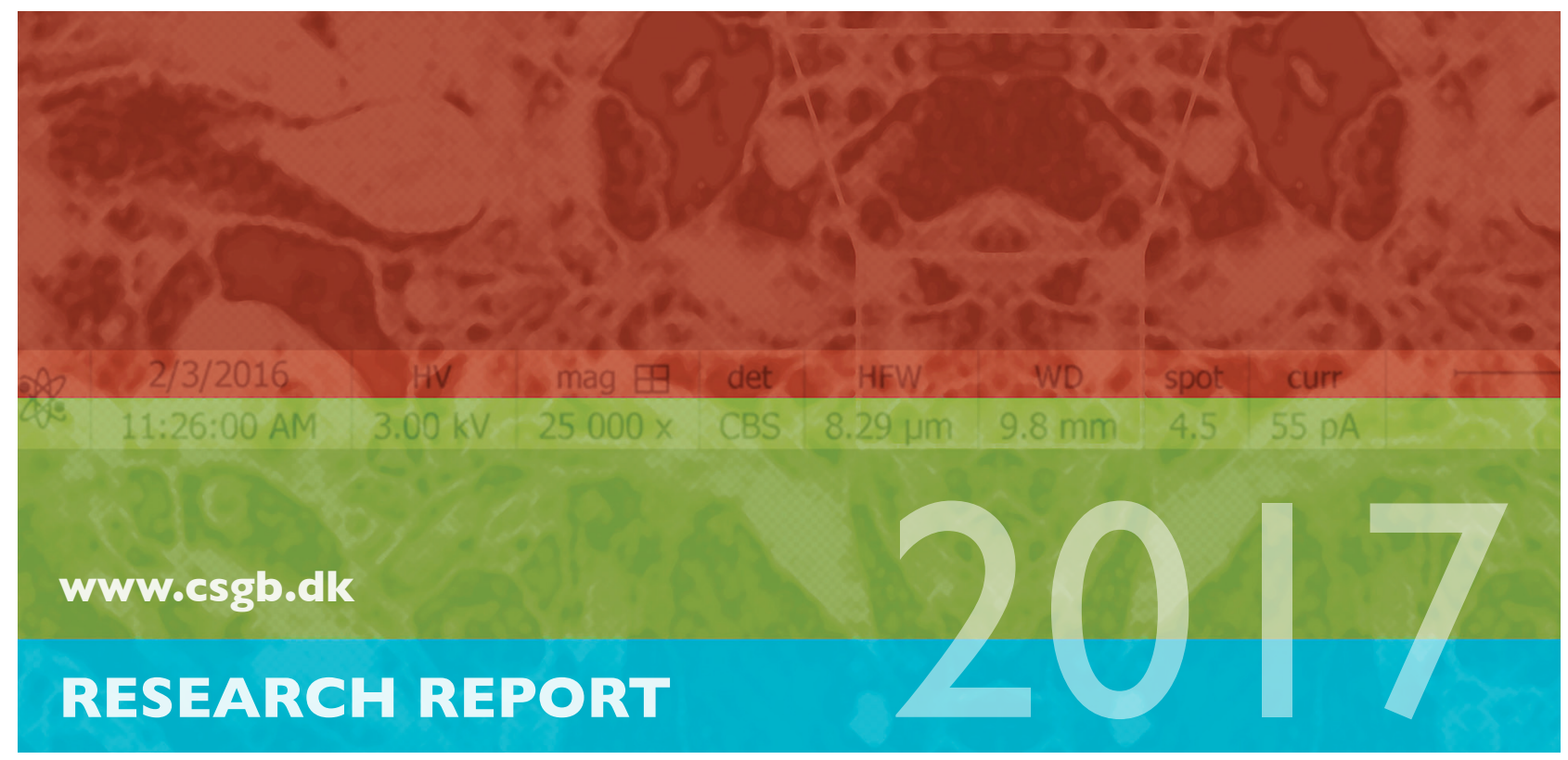

Alexis Arnaudon, Darryl D. Holm and Stefan Sommer

Stochastic metamorphosis with template uncertainties

No. 13, November 2017 


\title{
Stochastic metamorphosis with template uncertainties
}

\author{
Alexis Arnaudon ${ }^{1}$, Darryl D. Holm ${ }^{1}$ and Stefan Sommer ${ }^{2}$ \\ ${ }^{1}$ Department of Mathematics, Imperial College, London \\ ${ }^{2}$ Department of Computer Science (DIKU), University of Copenhagen
}

\begin{abstract}
In this paper, we investigate two stochastic perturbations of the metamorphosis equations of image analysis, in the geometrical context of the EulerPoincare theory. In the metamorphosis of images, the Lie group of diffeomorphisms deforms a template image that is undergoing its own internal dynamics as it deforms. This type of deformation allows more freedom for image matching and has analogies with complex fluids when the template properties are regarded as order parameters (coset spaces of broken symmetries). The first stochastic perturbation we consider corresponds to uncertainty due to random errors in the reconstruction of the deformation map from its vector field. We also consider a second stochastic perturbation, which compounds the uncertainty in of the deformation map with the uncertainty in the reconstruction of the template position from its velocity field. We apply this general geometric theory to several classical examples, including landmarks, images, and closed curves, and we discuss its use for functional data analysis.
\end{abstract}

\section{Introduction}

Variability in shapes can be modelled using flows of the group $G$ of diffeomorphic deformations of the ambient domain $\Omega$ in which the shape is embedded. This is the basis of the large deformation diffeomorphic metric mapping (LDDMM) framework, see Trouvé (1995); Christensen et al. (1996); Dupuis et al. (1998); Beg et al. (2005). In the LDDMM approach, the shape of an embedded template image $\eta \in N$ in the manifold of embedded shapes $\operatorname{Emb}(N, \Omega)$ changes via the action $g_{t} . \eta$ of time-dependent diffeomorphisms $g_{t} \in G$ on $\eta \in N$, through the action of $g_{t}$ on the domain $\Omega$. The metamorphosis extension Holm et al. (2009); Miller and Younes (2001); Trouvé and Younes $(2005 \mathrm{a}, \mathrm{b})$ of LDDMM introduces a further time-dependent variation $\eta_{t}$ of the template to model the combined dynamics $g_{t} \cdot \eta_{t}$.

In this paper, we combine the geometrical metamorphosis framework of Holm et al. (2009) with recent developments in stochastically perturbed Euler-Poincaré dynamics in fluid dynamics and shape analysis Holm (2015); Arnaudon et al. (2017, 2016), to model evolutions of both shape and template under stochastic perturbations. The resulting framework allows modelling of random evolutions of shape and 
template simultaneously. A potential application of such an evolution is in modelling the progression of disease using computational anatomy, in which the model would address the analysis of disease progression in both the population average and in the individual. From longitudinal image data, mean evolutions over the population can be inferred. While average template evolutions can be modelled deterministically, models for the dynamics of each individual subject that include stochastic uncertainty are arguably more realistic than models supporting only smooth deterministic trajectories. The stochastic metamorphosis model includes such non-smooth and non-deterministic variations by incorporating stochastic perturbations in shape and template simultaneously. We detail this application and outline further areas of applications where similar generative models of data appear, particularly in the combined modelling of phase and amplitude variation in functional data analysis.

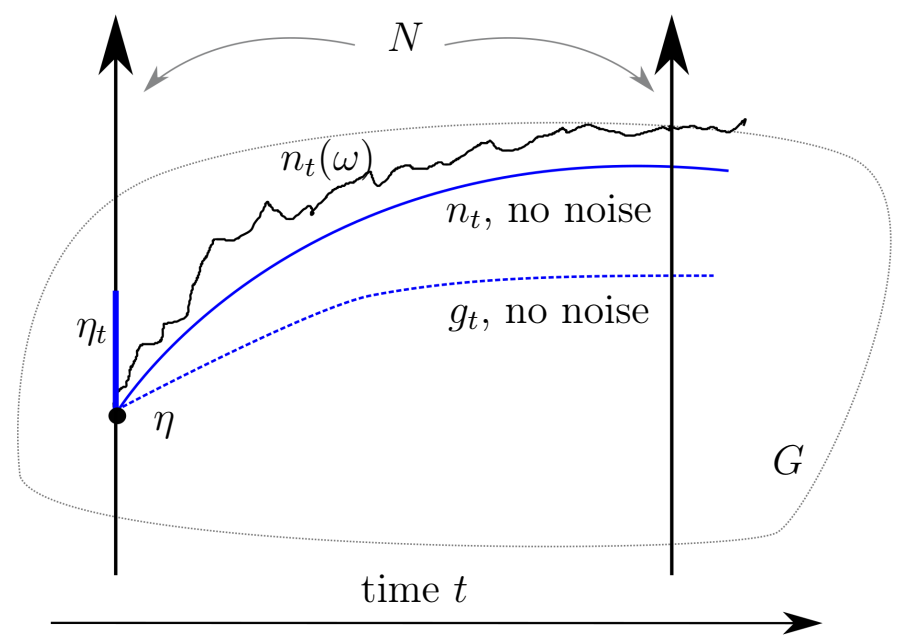

Figure 1: Sketch of the deformation group $G$, the shape space $N$ (vertical arrows), evolution of the deformation variable $g_{t}$, template variable $\eta_{t}$, and shape variable $n_{t}$ without noise $\left(W_{t}=0\right.$, blue), and shape variable $n_{t}$ with noise $\omega$ (black). The shape space is illustrated as being linear (e.g. landmarks, images). However, the framework applies to general non-linear shape spaces (e.g. curves, tensor fields).

\section{$1.1 \quad$ Background}

The LDDMM framework models the change of a shape $\eta \in N$ by the action of time dependent flows of diffeomorphisms $g_{t} \in G$ on the embedding space $\Omega$. One lifts the shape trajectory to a time-dependent curve $g_{t}$ on the diffeomorphisms by setting $n_{t}=g_{t} . \eta \in N$. For a right invariant metric on the tangent space of a subgroup $G$ of the diffeomorphism group $\operatorname{Diff}(N), N$ being the shape space, an energy can be defined as $E\left(g_{t}\right)=\int_{0}^{1}\left\|\partial_{t} g_{t}\right\|_{g_{t}}^{2} \mathrm{~d} t$. Combined with a data attachment term, this approach allows matching of shapes and image registration Beg et al. (2005). The invariance of $E\left(g_{t}\right)$ under the right action of $G$ implies that the metric descends to a metric structure on the data space $N$ itself. The action of $g_{t}$ differs between data types, but otherwise, the framework is formally equivalent for different classes of shapes. The use of the flows $g_{t}$ to model the shape variability is fundamental and the right trivialization $v_{t}:=\partial_{t} g_{t} \circ g_{t}^{-1}$ gives an Eulerian interpretation of the metric. 
The right invariance of the metric enables Euler-Poincare reduction of the dynamics to the Lie algebra of $G$ to be performed, and the critical paths for $E$ appear from the reduced dynamics.

Metamorphosis extends the LDDMM setting by letting the template vary in time as well as the deformation, thereby resulting in the flow $g_{t} \cdot \eta_{t}$, in which $\eta_{0}=\eta$ is the original template. The metamorphosis energy is encoded into a Lagrangian depending on both the $G$ and $N$ variability, again assuming invariance of the energy to the group action on both $G$ and $N$. A particular example of metamorphosis dynamics arises in image analysis, where the image $I_{t}$ changes both by deformation via the right action $g_{t} \cdot I_{t}=I_{t} \circ g_{t}^{-1}$ and via a pointwise change $\partial_{t} I_{t}(x)$ for each pixel/voxel $x$.

In this formulation of metamorphosis dynamics, an analogy with the flows of complex fluids arises. In complex fluids, a diffeomorphic flow carries an order parameter, defined as a coset space for a broken symmetry of homogeneous fluids, on which the diffeomorphisms act. The order parameter moves with the fluid, but it can also have its own internal dynamics, which in turn is coupled to the fluid motion Holm (2002); Gay-Balmaz and Ratiu (2009). A similar combined dynamics of shape and template also appears in the Fshape framework Charlier et al. (2017).

In Arnaudon et al. (2017, 2016), a stochastic model of shape evolution was introduced that preserves the Euler-Poincaré theory of the deterministic LDDMM framework. The model is based on the stochastic fluid dynamics model Holm (2015) where right-invariant noise is introduced to perturb the reconstruction equation that evolves the flow from the reduced dynamics. In deterministic LDDMM, the reconstruction equation specifies the evolution of the group element by $\partial_{t} g_{t}=v_{t} \circ g_{t}$ generated by the reduced Eulerian velocity vector field $v_{t}$. Stochasticity is introduced as a perturbation to the reconstruction equation, by introducing the stochastic time differential

$$
\mathrm{d} g_{t} g_{t}^{-1}=v_{t} \mathrm{~d} t+\sum_{l=1}^{N} \sigma_{l} \circ \mathrm{d} W_{t}^{l} .
$$

Here $W_{t}^{l}$ are standard Wiener processes and $\sigma_{l}$ are vector fields on the data domain which characterize the spatial correlation of the noise. As it turns out, the noise in Stratonovich form is denoted conventionally with the same symbol (o) that denotes composition of maps. This coincidence should not cause any confusion. However, just to be sure, we will write composition of maps as concatenation whenever the two meanings appear in the same equation, as in (1.1). The perturbation of the reduced variable implies that the noise is right-invariant and in a certain sense compatible with the right-invariant LDDMM metric. This approach preserves many of the geometric structures of the deterministic framework. Importantly, the descent of the stochastic model to particular data types is similar to the way the metric descends in the deterministic LDDMM framework.

A stochastic metamorphosis extension of the stochastic Euler-Poincaré framework was introduced in Holm (2017). The stochastic perturbations there were also introduced in the reduced variable influencing the deformation flow from the reconstruction equation. The template evolution $\eta_{t}$ is still deterministic. The aim of the present paper is to extend this model to include noise in the template evolution $\eta_{t}$ as well. We will make this extension on the reduced template velocity $g_{t} \partial_{t} \eta_{t}$ similarly 
to the perturbation of the group variable. This procedure results in simultaneous stochastic perturbations of the flow equations for both $g_{t}$ and $\eta_{t}$.

\subsection{Paper outline}

After a brief survey of the deterministic metamorphosis framework in section 2.1, we formally derive the stochastic model in section 2.2. We then show in section 2.3 how to derive these equations in the Hamilton-Pontryagin formulation, where the noise appears as a stochastic constraint in the variational principle. We end the theoretical section by deriving the corresponding Hamiltonian stochastic equations in 2.4 to then move to some classical examples of image analysis and computational anatomy in section 3, including landmarks and images. The inclusion of two types of stochastic variations links the framework to combined random phase and amplitude variations in functional data analysis. We provide perspectives of the method to future applications in functional data analysis and computational anatomy in section 4 .

\section{General stochastic metamorphosis}

In this section, we introduce the stochastic deformation of metamorphosis, but first, we recall the basis of this theory, in the context of reduction by symmetry. We will only review what will be needed for our exposition, and we refer to Holm et al. (2009) and Holm (2017) for more extensive treatments.

\subsection{Deterministic metamorphosis}

The theory of metamorphosis begins with a template $N$, considered here as a manifold (landmarks, images, etc. . ) upon which a group of diffeomorphism $G=\operatorname{Diff}(N)$ acts. The parameter space of this theory is $G \times N$, with curves $\left(g_{t}, \eta_{t}\right) \in G \times N$, where $g_{t}$ is the deformation curve and $\eta_{t}$ is the template curve. The image curve will be denoted $n_{t}=g_{t} \cdot \eta_{t} \in N$, where the dot represents the group action. This curve is the total motion of the template, or image $N$, under both the deformation and its own dynamics. For standard LDDMM, the motion of the image is only $n_{t}=g_{t} . \eta$, for a fixed reference template $\eta$. This combined action thus allows more freedom in the matching procedure, while remaining compatible with the theory of reduction by symmetries, which we now describe. We first define the two reduced velocity fields

$$
u_{t}:=\dot{g}_{t} g_{t}^{-1}, \quad \text { and } \quad \nu_{t}:=g_{t} \dot{\eta}_{t} .
$$

The first is the reduced deformation velocity and the second is the reduced template velocity. We then assume that the original Lagrangian of this theory is invariant under the group action of $G$, so that we may write the reduced Lagrangian in terms of the reduced velocity fields and the image position $n_{t}$, i.e.,

$$
L\left(g_{t}, \dot{g}_{t}, \eta_{t}, \dot{\eta}_{t}\right)=l\left(u_{t}, n_{t}, \nu_{t}\right) \text {. }
$$


Because the reduced Lagrangian still depends on the template variable $n_{t}$, reduction by the action of the diffeomorphisms will result in a semi-direct product structure, where the template is an advected quantity, in the language of fluid dynamics.

We next compute the variations of the three variables in the reduced Lagrangian, upon introducing the notation $\xi_{t}=\delta g_{t} g_{t}^{-1}$ and $\omega=g \delta \eta$, where $\delta g$ and $\delta \eta$ are free variations, to obtain

$$
\begin{aligned}
\delta u & =\dot{\xi}_{t}-\left[u_{t}, \xi_{t}\right], \\
\delta n & =\omega_{t}+\xi_{t} \eta_{t}, \\
\delta \nu & =\dot{\omega}_{t}+\xi_{t} \nu_{t}-u_{t} \omega_{t} .
\end{aligned}
$$

In these formulas, we need to specify what we mean by the multiplication, as the vector fields live in different spaces. In fact, $u_{t}, \xi_{t} \in \mathfrak{g}=\mathfrak{X}(N)$ are vector fields; so the Lie bracket is the natural operation. Recall that $\eta_{t} \in N$, thus $\xi_{t} \eta_{t}$ corresponds to the tangent map of the action of $G$ on the manifold $N$, and similarly for $\nu \in T N$, where the action is on the tangent space of $N$. We do not need these actions explicitly now, but we will need their 'adjoint action' in the following sense:

$$
\begin{aligned}
\left\langle n^{*} \diamond m, u\right\rangle_{\mathfrak{g}} & =-\left\langle n^{*}, u n\right\rangle_{N}, \\
\left\langle u \star \nu^{*}, \nu\right\rangle_{T N} & =\left\langle\nu^{*}, u \nu\right\rangle_{T N},
\end{aligned}
$$

where $N \in N, n^{*} \in N^{*}, \nu \in T N, \nu^{*} \in T^{*} N$ and $u \in \mathfrak{g}$. The first equality defines the diamond operation $(\diamond)$, which will serve as a force term to capture the coupling between the advected quantity $n$ and the main dynamics of the diffeomorphism group. The second equality defines the star operation $(\star)$, which is the adjoint of the action of $u$ on $T N$. That is, it defines the action of $u$ on $T^{*} N$.

Applying the variational calculus to the action $S=\int l \mathrm{~d} t$, we obtain the EulerPoincaré formulation of the metamorphosis equation in the form

$$
\begin{aligned}
& \frac{\mathrm{d}}{\mathrm{d} t} \frac{\delta l}{\delta u}+\operatorname{ad}_{u_{t}}^{*} \frac{\delta l}{\delta u}+\frac{\delta l}{\delta n} \diamond n+\frac{\delta l}{\delta \nu} \diamond \nu=0, \\
& \frac{\mathrm{d}}{\mathrm{d} t} \frac{\delta l}{\delta \nu}+u_{t} \star \frac{\delta l}{\delta \nu}-\frac{\delta l}{\delta n}=0
\end{aligned}
$$

together with the reconstruction equation

$$
\dot{n}=u_{t} n_{t}+\nu_{t}
$$

We refer to Holm et al. (2009); Holm (2017) for the details of this derivation, which we will do in the context of Hamilton-Pontryagin with noise in the next section.

From here, a choice of Lagrangian and data $N$ will reduce the system to particular cases, some of which we discuss in the applications section 3.

\subsection{Formal derivation of the stochastic equations}

We will first derive the equation informally, using 'stochastic variations', then show a more straightforward derivation using the Hamilton-Pontryagin principle. The second derivation also has the advantage of revealing the effects of the noise more transparently. 
In order to introduce a noise compatible with the Euler-Poincaré equation, we need to perturb the theory at its core, which is in this case the definition of the reduced velocities in (2.1). Indeed, the variations were computed from these definitions, and the deterministic Euler-Poincaré equation emerged. Although a single relation is used in the Euler-Poincaré equation (2.7), we will split it into two parts, and perturb them with two different noise components as follows,

$$
\begin{aligned}
\mathrm{d} g_{t} g_{t}^{-1} & =u_{t}(x) \mathrm{d} t+\sum_{l=1}^{K^{u}} \sigma_{l}^{u}(x) \circ \mathrm{d} W_{t}^{l}=: \mathrm{d} u_{t}(x), \\
g_{t} \mathrm{~d} \eta & =\nu_{t} \mathrm{~d} t+\sum_{k=1}^{K^{\nu}} \sigma_{k}^{\nu} \circ \mathrm{d} W_{t}^{k}=: \mathrm{d} \nu_{t} .
\end{aligned}
$$

In a slight abuse of notation, $\mathrm{d} u_{t}(x)$ and $\mathrm{d} \nu_{t}$ are written as stochastic processes. Here $\sigma_{l}^{u}: N \rightarrow \mathfrak{g}$ are a set of $K^{u}$ vector fields on the domain $\Omega$, and $\sigma_{l}^{\nu} \in T N$ are another set of $K^{\nu}$ tangent vectors on the template. We also denote by $W_{t}^{l}$ or $W_{t}^{k}$ the $K^{u}+N^{\nu}$ independent standard Weiner processes. In addition, we denote by $x_{0} \in \Omega$ the Lagrangian labels upon which $g_{t}$ acts, so that the first equation can be written equivalently as

$$
\mathrm{d} g_{t}=u_{t}\left(g_{t} x_{0}\right) \mathrm{d} t+\sum_{l=1}^{K^{u}} \sigma_{l}^{u}\left(g_{t} x_{0}\right) \circ \mathrm{d} W_{t}^{l} .
$$

The second equation for $\eta$ in (2.8) does not have any $x_{0}$ dependence, as it is an equation for the template itself. Thus, $\sigma_{k}^{\nu}$ are not functions of $N$; rather, they are tangent vectors to $N$.

With the notation for $\mathrm{d} u_{t}$ and $\mathrm{d} \nu_{t}$ in (2.8), we have the complete reconstruction relation for the stochastic image template $n_{t}$

$$
\mathrm{d} n_{t}=\mathrm{d} u_{t} n_{t}+\mathrm{d} \nu_{t} .
$$

Because $n_{t} \in N$, the concatination $\mathrm{d} u_{t} n_{t}$ means the composition $\mathrm{d} u_{t}\left(n_{t}\right)$. In (2.9), the noise in the $u_{t}$ vector field was introduced in Holm (2017), based on the stochastic fluid dynamics model of Holm (2015), whereas the noise in the $\nu_{t}$ field is new. The first noise term in (2.9) corresponds to random errors in the reconstruction of the diffeomorphism path from its velocity field, while the second one represents random errors for the reconstruction of the template position from its velocity field. In stochastic metamorphosis, the two noise terms will affect the dynamical equations differently.

From these stochastic perturbations of the reconstruction relation, we can formally compute the variations and obtain

$$
\begin{aligned}
& \delta u=\mathrm{d} \xi_{t}+\left[\xi, \mathrm{d} u_{t}\right], \\
& \delta \nu=\mathrm{d} \omega+\xi \mathrm{d} \nu_{t}-\mathrm{d} u_{t} \omega .
\end{aligned}
$$

These are convenient expressions, but they introduce the variations as stochastic processes; so they should not be taken at face value without further analysis. We will see in the next section how to re-derive these equations without introducing 
stochastic variations, by using the Hamilton-Pontryagin principle. Because the results are identical for the two methods, we can proceed formally here by using these variations as we did in the deterministic variational principle to obtain the following stochastic reduced metamorphosis equations in Euler-Poincaré form,

$$
\begin{aligned}
& \mathrm{d} \frac{\delta l}{\delta u}+\mathrm{ad}_{\mathrm{d} u_{t}}^{*} \frac{\delta l}{\delta u}+\frac{\delta l}{\delta n} \diamond n \mathrm{~d} t+\frac{\delta l}{\delta \nu} \diamond \mathrm{d} \nu_{t}=0, \\
& \mathrm{~d} \frac{\delta l}{\delta \nu}+\mathrm{d} u_{t} \star \frac{\delta l}{\delta \nu}-\frac{\delta l}{\delta n} \mathrm{~d} t=0,
\end{aligned}
$$

as well as equation (2.9), all to be compared with the deterministic case in equations (2.6) and (2.7).

\subsection{Derivation using the Hamilton-Pontryagin principle}

We now show how to rederive the stochastic metamorphosis equations more transparently, without introducing stochastic variations (2.10). For this purpose, we will use the stochastic Hamilton-Pontryagin approach and closely follow the exposition of Holm (2017).

The deterministic Hamilton-Pontryagin principle is a variational principle with the following constrained action

$$
\begin{aligned}
S\left(u_{t}, n_{t}, \dot{n}_{t}, \nu_{t}, g_{t}, \dot{g}_{t}\right)=\int_{0}^{1} l\left(u_{t}, n_{t}, \nu_{t}\right) \mathrm{d} t & +\int_{0}^{1}\left\langle M_{t},\left(\dot{g}_{t} g_{t}^{-1}-u_{t}\right)\right\rangle \mathrm{d} t \\
& +\int_{0}^{1}\left\langle\sigma_{t},\left(\dot{n}_{t}-\nu_{t}-u_{t} n_{t}\right)\right\rangle \mathrm{d} t,
\end{aligned}
$$

where $M_{t} \in \mathfrak{X}^{*}(N)$ and $\sigma_{t} \in T^{*} N$ are generalised Lagrange multipliers to enforce the constraint of the reconstruction relations. Taking free variations for all the variables yields the deterministic reconstruction relation (2.7) and the deterministic EulerPoincaré equation (2.6). We refer to Holm (2017) for more details of the derivation. The crucial point here is to allow free variations, by introducing constraints into the variational principle, and not in the variations as in the standard Euler-Poincaré reduction theory. An alternative approach would be to use the Clebsch constrained variational method used for fluid dynamics in Holm (2015).

In the present context, we enforce the stochastic reconstruction relations (2.8) via the following stochastic Hamilton-Pontryagin principle

$$
\begin{aligned}
S\left(u_{t}, n_{t}, \mathrm{~d} n_{t}, \nu_{t}, g_{t}, d g_{t}\right)=\int_{0}^{1} l\left(u_{t}, n_{t}, \nu_{t}\right) & +\int_{0}^{1}\left\langle M_{t},\left(\mathrm{~d} g_{t} g_{t}^{-1}-\mathrm{d} u_{t}\right)\right\rangle \\
& +\int_{0}^{1}\left\langle\sigma_{t},\left(\mathrm{~d} n_{t}-\mathrm{d} \nu_{t}-\mathrm{d} u_{t} n_{t}\right)\right\rangle
\end{aligned}
$$


or, more explicitly, upon substituting for $\mathrm{d} u_{t}$ and $\mathrm{d} \nu_{t}$ from (2.8), we have

$$
\begin{aligned}
S\left(u_{t}, n_{t}, \mathrm{~d} n_{t}, \nu_{t}, g_{t}, \mathrm{~d} g_{t}\right)= & \int_{0}^{1} l\left(u_{t}, n_{t}, \nu_{t}\right) \mathrm{d} t \\
& +\int_{0}^{1}\left\langle M_{t}, \mathrm{~d} g_{t} g_{t}^{-1}-u_{t} \mathrm{~d} t-\sum_{l=1}^{K^{u}} \sigma_{l}^{u}(x) \circ \mathrm{d} W_{t}^{l}\right\rangle \\
& +\int_{0}^{1}\left\langle\sigma_{t}, \mathrm{~d} n_{t}-\nu_{t} \mathrm{~d} t-\sum_{k=1}^{K^{\nu}} \sigma_{k}^{\nu}(x) \circ \mathrm{d} W_{t}^{k}\right\rangle \\
& -\int_{0}^{1}\left\langle\sigma_{t},\left(u_{t} \mathrm{~d} t+\sum_{l=1}^{K^{u}} \sigma_{l}^{u}(x) \circ \mathrm{d} W_{t}^{l}\right) n_{t}\right\rangle .
\end{aligned}
$$

Proposition 2.1. The stochastic variational principle $\delta S=0$ with action (2.14) yields the stochastic Euler-Poincaré equation (2.11) with stochastic reconstruction relation (2.8) and (2.9).

Proof. The proof is a direct computation by taking free variations. We will show the key steps below. First, the variations with respect to $M_{t}$ and $\sigma_{t}$ yield the reconstruction relations (2.8) and (2.9). Then, the variations with respect to $u_{t}, n_{t}$ and $\nu_{t}$ specify

$$
\frac{\delta l}{\delta u_{t}}=M_{t}+\sigma_{t} \diamond n_{t}, \quad \text { textand } \quad \frac{\delta l}{\delta \nu_{t}}=\sigma_{t} .
$$

We also have, for the $n_{t}$ variations,

$$
\frac{\delta l}{\delta n_{t}} \mathrm{~d} t=\mathrm{d} \sigma_{t}+u_{t} \star \sigma_{t} \mathrm{~d} t+\sum_{l=1}^{K^{u}} \sigma_{l}^{u}(x) \star \sigma_{t} \circ \mathrm{d} W_{t}^{l} .
$$

Finally, for $\xi=\delta g g^{-1}$ vanishing at the endpoints, we have

$$
\delta\left(\mathrm{d} g_{t} g_{t}^{-1}\right)=\mathrm{d} \xi-\left[u_{t} \mathrm{~d} t+\sum_{l=1}^{K^{u}} \sigma_{l}^{u}(x) \circ \mathrm{d} W_{t}^{l}, \xi\right] .
$$

From this computation, we have the last term in the calculus of variations which reads

$$
\mathrm{d} M_{t}=-\operatorname{ad}_{u_{t}}^{*} M_{t}-\sum_{l=1}^{K^{u}} \operatorname{ad}_{\sigma_{l}^{u}(x)}^{*} M_{t} \circ \mathrm{d} W_{t}^{l} .
$$

Finally, substituting the values of $M_{t}$ and $\sigma_{t}$ of (2.15) in equation (2.16) and (2.18) yields the stochastic metamorphosis equation (2.11) after a few more manipulations (see Corollary 3 of Holm (2017)).

\subsection{Hamiltonian formulation}

Provided that the Lagrangian is hyperregular, the stochastic metamorphosis equation (2.11) can be written as a stochastic Hamiltonian equation with Hamiltonian obtained via the reduced Legendre transform,

$$
h(\mu, \sigma, n)=\langle\mu, u\rangle+\langle\sigma, \nu\rangle-l(u, \nu, n),
$$


in which $\mu$ and $\sigma_{t}$ are the conjugate variables of $u_{t}$ and $\nu_{t}$, respectively. The noise is encoded into the stochastic potentials

$$
\Phi_{l}^{u}\left(\mu_{t}\right)=\left\langle\mu_{t}, \sigma_{l}^{u}\right\rangle_{\mathfrak{g} \times \mathfrak{g}^{*}}, \quad \text { and } \quad \Phi_{k}^{\nu}\left(\sigma_{t}\right)=\left\langle\sigma_{t}, \sigma_{k}^{\nu}\right\rangle_{T N \times T^{*} N},
$$

such that the stochastic equation of motion has a Hamiltonian drift term with $h$ and stochastic terms obtained via the same Hamiltonian structure, but with stochastic potentials. Notice that the two potentials have a different pairing, one on the Lie algebra of the diffeomorphism group, and the other on the tangent space of the template manifold. The Hamiltonian structure is given in Holm (2017) and we will only display here the Hamiltonian equations

$$
\begin{aligned}
\mathrm{d} \mu_{t} & +\operatorname{ad}_{\frac{\delta h}{\delta \mu}}^{*} u \mathrm{~d} d t+\sigma \diamond \frac{\delta h}{\delta \sigma} \mathrm{d} t+\frac{\delta h}{\delta n} \diamond n \mathrm{~d} t \\
& +\sum_{l} \operatorname{ad}_{\frac{\delta \Phi_{l}^{u}}{\delta \mu}}^{*} u \circ \mathrm{d} W_{t}^{l}+\sum_{l} \sigma_{t} \diamond \frac{\delta \Phi_{l}^{\nu}}{\delta \sigma} \circ \mathrm{d} W_{t}^{l}=0, \\
\mathrm{~d} \sigma_{t} & +\frac{\delta h}{\delta \mu} \star \sigma_{t} \mathrm{~d} t-\frac{\delta h}{\delta n}+\sum_{l} \frac{\delta \Phi_{l}^{u}}{\delta \mu} \star \sigma_{t} \circ \mathrm{d} W_{t}^{l}=0 .
\end{aligned}
$$

In the examples in the next section, we will use this formulation to derive the stochastic equations of motion. Taking the Hamiltonian approach turns out to be more transparent than the Lagrangian description.

\section{Applications}

Following Holm et al. (2009), we explicitly provide the stochastic metamorphosis equations for a few classical examples, including landmarks and images, and leave other applications such as closed planar curves, densities or tensor fields for later works.

\subsection{Landmarks and peakons}

Consider the case when the template manifold $N$ is the space of $n$ landmarks $\mathbf{q}=$ $\left(q_{1}, \ldots, q_{n}\right) \in \Omega^{n}$ with momenta $\mathbf{p}=\left(p_{1}, \ldots, p_{n}\right) \in T_{\mathbf{q}} \Omega^{n} \cong \Omega^{n}$. One needs to specify a Lagrangian for this system, and the simplest is

$$
l(u, n, \nu)=\frac{1}{2}\|u\|_{K}^{2}+\frac{\lambda^{2}}{2} \sum_{i=1}^{n}\left|p_{i}\right|^{2},
$$

where the first norm depends on the kernel $K(x)$ and the second norm is the vector norm of the momenta multiplied by a constant $\lambda^{2}$. In this case, we interpret the momenta as the conjugate variables to the template deformation vector field $\nu$ in order to have an equation only in term of the position and momenta of the landmarks. The derivation of the landmark equation is rather standard. Hence, we will only show it on the Hamiltonian side. We refer, for example, to Holm et al. (2009) for more details of the deterministic derivation, or to Arnaudon et al. (2017) and Holm and Tyranowski (2016) for discussions of the stochastic landmark dynamics. 
Recall that the landmark Hamiltonian is

$$
h_{K}(\mathbf{p}, \mathbf{q})=\frac{1}{2} \sum_{i j} p_{i} \cdot p_{j} K\left(q_{i}-q_{j}\right),
$$

and the metamorphosis Hamiltonian is thus

$$
h\left(q_{i}, p_{i}\right)=h_{K}(\mathbf{q}, \mathbf{p})+\frac{\lambda^{2}}{2} \sum_{i=1}^{n}\left|p_{i}\right|^{2} .
$$

The stochastic potentials $(2.20)$ become in this case

$$
\Phi_{l}^{u}(\mathbf{q}, \mathbf{p})=\sum_{i} p_{i} \cdot \sigma_{l}^{u}\left(q_{i}\right) \quad \text { and } \quad \Phi_{i}^{\nu}(\mathbf{p})=p_{i} \cdot \sigma_{i}^{\nu}
$$

Notice that the stochastic potential $\Phi^{\nu}$ is described by a fixed vector, where $\sigma_{i}^{\nu}$ is the amplitude of the noise for the landmark $i$. However, for the stochastic potential $\Phi^{u}$, we have to specify space (or $\mathbf{q}$ ) dependent functions $\sigma_{l}^{u}(\mathbf{q})$. This simple form comes from the fact that we used a discrete set of points and $\nu=\mathbf{p}$ for the template deformation, and the summation over $k$ becomes a summation over the landmark index. In addition, a sum of two Wiener process is another Wiener process with the sum of the amplitude (if it is additive and in Itô form). From this observation, one can see that the general equation $\Phi_{k}^{\nu}(\mathbf{p})=\sum_{i} p_{i} \cdot \sigma_{k}^{\nu}$ is equivalent to a change of amplitudes $\sigma_{k}^{\nu}$ and $i=k$.

We compute the stochastic Hamiltonian equations for landmarks to arrive at

$$
\begin{aligned}
\mathrm{d} q_{i} & =\frac{\partial h_{K}}{\partial p_{i}} \mathrm{~d} t+\sum_{l} \sigma_{l}^{u} \circ \mathrm{d} W_{t}^{l}+\lambda^{2} p_{i} \mathrm{~d} t+\sigma_{i}^{\nu} \mathrm{d} W_{t}^{i}, \\
\mathrm{~d} p_{i} & =-\frac{\partial h_{K}}{\partial q_{i}} \mathrm{~d} t+\sum_{l} \partial_{q_{i}}\left(p_{i} \cdot \sigma_{l}^{u}\right) \circ \mathrm{d} W_{t}^{l},
\end{aligned}
$$

in which we can use the Itô integral for the $\nu$-noise, as it is additive.

Notice that setting $\lambda=0$ recovers the standard landmark dynamics, but with an additive noise in the position equation. This is different from the conventional physical perspective, in which additive noise often appears in the momentum equation, as in Trouvé and Vialard (2012); Vialard (2013); Marsland and Shardlow (2017).

\section{$3.2 \quad$ Images}

The present stochastic metamorphosis framework can be directly applied to images, by taking the template space $N$ to be the space of smooth functions from the domain $\Omega \subset \mathbb{R}^{2}$ to $\mathbb{R}$. We set $u_{t} \in \mathfrak{X}(\Omega)$ the deformation vector field and $\rho \in T N \cong N$ the template vector field. As before, the Lagrangian must have two parts, and the simplest non-trivial one is the sum of kinetic energies written as

$$
l(u, n, \nu)=\frac{1}{2}\left\|u_{t}\right\|_{K}^{2}+\frac{\lambda^{2}}{2}\left|\rho_{t}\right|_{L^{2}}^{2},
$$


where the first norm depends on the kernel $K$ and the second norm is the standard $L^{2}$ norm over $\Omega$. By choosing a $L^{2}$ norm we can identify $\rho_{t}$ with its dual in the case $\lambda=1$. We will thus not distinguish between $\sigma_{t}$ and $\nu_{t}$ of the general framework.

Thus, as before, we use the Hamiltonian formulation of the stochastic metamorphosis equations with the stochastic potentials,

$$
\Phi_{l}^{u}\left(m_{t}\right)=\int_{\Omega}\left\langle m_{t}(x), \sigma_{l}^{u}(x)\right\rangle d x \quad \text { and } \quad \Phi_{k}^{\nu}\left(\sigma_{t}\right)=\int_{\Omega}\left\langle\rho_{t}(x), \sigma_{l}^{\nu}(x)\right\rangle d x .
$$

Notice that in this case, both $\sigma_{l}^{u}$ and $\sigma_{l}^{\nu}$ are functions of the domain $\Omega$, and they encode spatial correlation structure of the stochastic perturbations.

Then, because the Hamiltonian structure has three sorts of terms, the $\mathrm{ad}^{*}$, the $\diamond$ and the $\star$ terms defined in equation $(2.5)$, which in this case are

$$
\begin{aligned}
\operatorname{ad}_{u_{t}}^{*} m_{t} & =\left(u_{t} \cdot \nabla\right) m_{t}+\left(m_{t} \cdot \nabla\right) u_{t}+\operatorname{div}\left(u_{t}\right) m_{t}, \\
\sigma_{t} \diamond \nu_{t} & =\sigma_{t} \cdot \nabla \nu_{t}, \\
u_{t} \star \sigma_{t} & =\nabla \cdot\left(\sigma_{t} u_{t}\right),
\end{aligned}
$$

we arrive at the following set of stochastic PDEs (for any $\lambda$ )

$$
\begin{aligned}
& \mathrm{d} m_{t}+\operatorname{ad}_{u_{t}}^{*} m_{t} \mathrm{~d} t+\sum_{l} \operatorname{ad}_{\sigma_{l}^{u}}^{*} m_{t} \circ \mathrm{d} W_{t}^{l}=\lambda^{2} \rho_{t} \cdot \nabla \rho_{t} \mathrm{~d} t+\sum_{k} \rho_{t} \cdot \nabla \sigma_{k}^{\nu} \circ \mathrm{d} W_{t}^{k}, \\
& \mathrm{~d} \rho_{t}+\nabla \cdot\left(\rho_{t} u_{t}\right) \mathrm{d} t+\sum_{l} \nabla \cdot\left(\rho \sigma_{l}^{u}\right) \circ \mathrm{d} W_{t}^{l}=0 .
\end{aligned}
$$

Another important equation is the reconstruction relation (2.9), which now reads

$$
\mathrm{d} g_{t}=u_{t}\left(g_{t}\right) \mathrm{d} t+\sum_{l} \sigma_{l}^{u}\left(g_{t}\right) \circ \mathrm{d} W_{t}^{l}+\rho_{t} \mathrm{~d} t+\sum_{k} \sigma_{k}^{\nu} \circ \mathrm{d} W_{t}^{k} .
$$

Notice that if we set $\lambda=1$, the effect of the density, or template motion on the momentum $m$ only appears via the noise term, similarly to the landmark case.

In the one dimensional case, the metamorphosis equation is known to reduce to the so-called $\mathrm{CH} 2$ system, which is equation coupling the Camassa-Holm equation with a density advection equation for $\rho_{t}=\nu_{t}$. We refer to Holm et al. (2009); Chen et al. (2006) and references therein for more details about this equation and its complete integrability in the deterministic case. A similar reduction holds for both stochastic deformations, and we have the following stochastic $\mathrm{CH} 2$ equation

$$
\begin{aligned}
\mathrm{d} m & +\left(u \partial_{x} m+2 m \partial_{x} u\right) \mathrm{d} t \\
& =-\rho \partial_{x} \rho \mathrm{d} t-\sum_{k} \rho \partial_{x} \sigma_{k}^{\nu} \circ \mathrm{d} W_{t}^{k}-\sum_{l}\left(\sigma_{l}^{u} \partial_{x} m+\sum_{l} 2 m \partial_{x} \sigma_{l}^{u}\right) \circ \mathrm{d} W_{t}^{l}, \\
\mathrm{~d} \rho & +\partial_{x}(\rho u) \mathrm{d} t+\partial_{x}\left(\rho \sigma_{l}^{u}\right) \circ \mathrm{d} W_{t}^{l}=0 .
\end{aligned}
$$

Compared to the landmark example, the noise associated to the template dynamics is described by a set of functions of the image, not a set of fixed vectors. The difference between the nature of these two types of noise is thus less apparent, apart from how they appear in the equation. 


\section{Perspectives}

\subsection{Computational Anatomy}

Estimation of population atlases and longitudinal analysis of anatomical changes caused by disease progression constitute integral parts of computational anatomy Younes et al. (2009). The relation between these problems and the stochastic metamorphosis model presented here can be illustrated by the analysis of longitudinal brain MR-image data of patients suffering from Alzheimer's disease. The data manifold $N$ is here a vector space of images as described above with $\Omega \subseteq \mathbb{R}^{3}$.

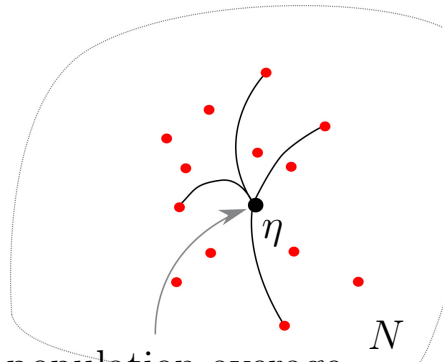

population average
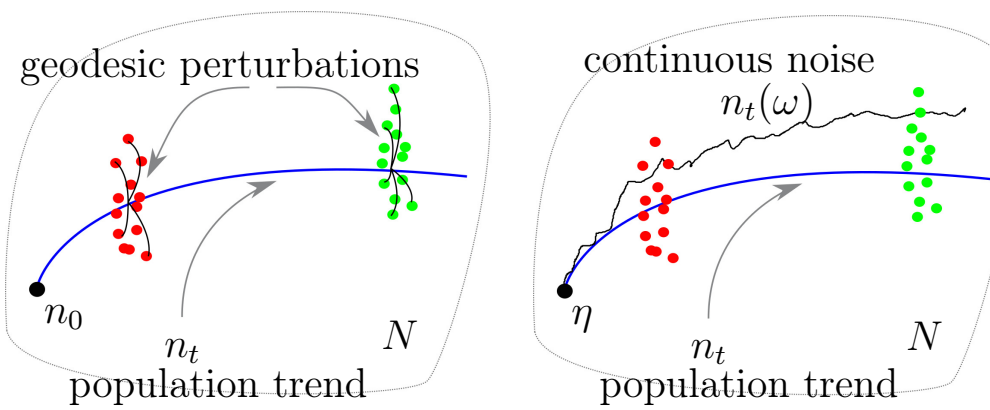

Figure 2: (left) Template estimation in the form (4.1) aims at finding a single descriptor $\eta$ for the population average of the observed shapes $n^{1}, \ldots, n^{k}$ (red dots) in the non-linear shape space $N$. The variational principle (4.1) corresponds to assuming $n^{i}$ arise from geodesic perturbations of $\eta$. (center) Geodesic regression models a population trend as a geodesic $n_{t}$. Observations at different time points $\left(n_{t_{1}}^{i}\right.$ red, $n_{t_{2}}^{i}$ green) arise as perturbations of the points $n_{t_{1}}$ and $n_{t_{2}}$ by random geodesics. (right) Stochastic metamorphosis models the evolution of the population trend $n_{t}$ deterministically while observations $n_{t_{j}}^{i}=n_{t_{j}}\left(\omega^{i}\right)$ appear from individual noise realizations $\omega^{i}$. The perturbations are time continuous and apply to each case $i$ individually making the model natural for modelling longitudinal evolutions with noise.

Focusing first on template estimation, in medical imaging commonly denoted atlas estimation, the aim is to find a population average of data assumed observed at a fixed time point. In the literature, this is for example pursued by minimizing the total sum of the regularized LDDMM energies of deterministic geodesic trajectories that deform the atlas to match the observed data Joshi et al. (2004). For $k$ data points $n^{1}, \ldots, n^{k}$ and with data matching term $S: N \times N \rightarrow \mathbb{R}$, the template $\eta$ is then estimated by joint minimization of

$$
\min _{\left(\eta, v_{t}^{1}, \ldots, v_{t}^{k}\right)} \sum_{i=1}^{k} \int_{0}^{1}\left\|v_{t}^{i}\right\|^{2} \mathrm{~d} t+S\left(\phi_{T}^{i} \cdot \eta, n^{i}\right),
$$

where the deformations $\phi_{T}^{i}$ each are endpoints of the integral of the vector fields $v_{t}^{i}$ on an interval $[0, T]$.

A different approach to atlas estimation is to perform inference in statistical models where observations are assumed random perturbations of a template and inference of the template is performed via maximum-likelihood or maximum-a-posteriori 


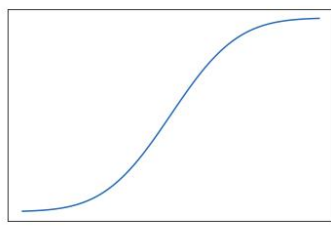

(a) tempate signal $\eta$

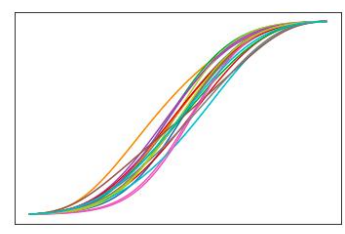

(b) phase variation $(\phi)$

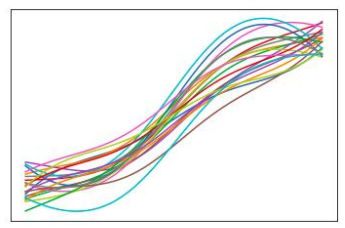

(c) amplitude variation $(\nu)$

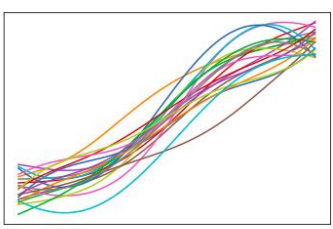

(d) phase and amplitude variation

Figure 3: A template signal (a) can be perturbed by (b) variation in phase, in (4.2) denoted $\phi$; (c) variation in amplitude, $\nu$ in (4.2); (d) phase and amplitude simultaneously.

estimation. This approach is pursued, for example, in a Allassonnière et al. (2007); Zhang et al. (2013); Pai et al. (2016). See also the discussion below.

Longitudinal analysis aims at capturing the average time evolution of the brain shape caused by the disease Muralidharan and Fletcher (2012); Niethammer et al. (2011). A common approach here is to estimate a general deterministic trend that is perturbed by noise at discretely observed time points in order to describe the observed images Fletcher and Zhang (2016). For example, the noise can take the form of random initial velocity vectors for geodesics emanating from the deterministic trajectory.

The stochastic metamorphosis framework proposed here combines deterministic longitudinal evolution of the template in both shape, represented by the deformations $g_{t}$, and in the template image, $n_{t}=g_{t} \cdot \eta_{t}$. We can assume longitudinal observations $n_{t_{j}}^{i}, i=1, \ldots, k, j=1, \ldots, t_{l}$ at $l$ time points are realizations of the stochastic model with time-continuous noise process drawn for each subject $i$. The stochastic perturbations are thus tied to each subject affecting the dynamics simultaneously with the evolution of the deterministic flow. The relation between this model, geodesic regression models, and atlas estimation is illustrated in Figure 2.

Because of the randomness, algorithms for inference of the template $\eta$ and its evolution $n_{t}=g_{t} . \eta_{t}$ from data can naturally be formulated by matching statistics of the data, e.g. by matching moments or by maximum-likelihood as done for the landmark case of stochastic EPDiff equations in Arnaudon et al. (2017). Development of such inference schemes constitutes natural future research directions.

\subsection{Phase and Amplitude in Functional Data Analysis}

While images exhibit variations in both intensity and shape of the image domain, signals in functional data analysis often exhibit combined variation in amplitude and phase. For a signal $f: I \rightarrow N$ defined on an interval $I$, amplitude variations refer to variations of the values $f(s)$ in $N$ for each fixed $s \in I$ while phase variation covers changes in the parametrization of the domain $I$. This is illustrated with $N=\mathbb{R}$ in Figure 3. An example of such combined phase and amplitude variations is provided in the growth curves of children and young adults; in which phase variation is connected to the absolute height of the subject while phase variation arise from growth and growth spurts occurring at different ages for different children. 
Recent literature covers multiple approaches for identifying, separating and performing inference in situations with combined phase and amplitude variation Raket et al. (2014); Marron et al. (2015); Tucker et al. (2013). One example of a generative model in this settings is the mixed-effects model Raket et al. (2014); Kühnel et al. (2017)

$$
f(s)=\eta\left(\phi^{-1}(s)\right)+\nu(s)+\epsilon, s \in I,
$$

where the average signal $\eta$ is deformed in phase by the action $\phi . \eta=\eta \circ \phi^{-1}$ of a deformation $\phi$ of the interval $I$, and in amplitude by the additive term $\nu$. Here $\eta$ is considered a fixed, non-random effect while both $\phi$ and $\nu$ are random. Illustrated with the growth curve case above, $\eta$ models the population average growth curve for each age $s$, while $\phi$ controls the timing of the growth process for the individual children and $\nu$ the absolute height difference to the population average. One observes that the model (4.2) is non-linear, because of the coupling between $\phi$ and $\eta$. In addition, a model for the deformations $\phi$ is needed, and the randomness appearing in both $\phi$ and $\nu$ must be specified.

Whereas the LDDMM model is widely used in image analysis, this framework has not yet seen many applications for modelling deformations in functional data analysis, such as the phase variation appearing in (4.2). Instead, works such as Raket et al. (2014) use a small-deformation model $\phi(s)=s+v(s)$ with random vector field $v$ modelling displacements on $I$. On the other hand, large-deformation flow models such as LDDMM traditionally have not integrated random variation directly into the dynamics. Natural families of probability distributions and generative models taking values in non-linear spaces such as deformation spaces are generally non-trivial to construct. However, the model proposed in this paper achieves exactly that.

A direct metamorphosis equivalent of the mixed-effects model (4.2) has $\eta=\eta_{0}$ the population average $\eta$, sets $u_{0}=\nu_{0}=0$ and encodes the random effects $\phi$ and $\nu$ in (4.2) in the stochastic increments $d u_{t}$ and $d \nu_{t}$. The action of $g_{t}$ on the signal is the right action $g_{t} . f=f \circ g_{t}^{-1}$ as in (4.2). Now $d \nu_{t}$ models pure amplitude variation, $d u_{t}$ phase variation, and the combined stochastic evolution of the signal is $d f_{t}=d n_{t}$. We then assume the observed signal is $f=f_{T}$ for a fixed end time $T$ of the stochastic process. Spatial correlation in both the deformation increments $d u_{t}$ and the amplitude increments $d \nu_{t}$ is encoded in the fields $\sigma_{l}^{u}$ and $\sigma_{k}^{\nu}$ respectively.

In the above model, the template is stationary in time when disregarding the stochasticity. However, allowing non-zero initial momenta $u_{0}$ and $\nu_{0}$ in both phase and amplitude allows the template to vary with time and thereby gives a non-linear generalization of a standard multivariate regression model with one latent variable for phase and one for amplitude. This in particular allows modelling of trends over populations where subjects are affected by both the population trend and individual stochastic perturbations.

\subsection{Statistical nonlinear modelling}

It may initially seem overly complicated to use the metamorphosis framework for a simple regression model. However, statistical models that in linear space seem completely standard are often inherently difficult to generalize to non-linear spaces. In general, the lack of vector space structure makes distributions and generative models 
hard to specify, see e.g. Sommer (2015); Sommer and Svane (2017) for examples of the geometric complexities of generalizing the Euclidean normal distribution.

In Euclidean space, random vectors can model random perturbations. In nonlinear spaces, the lack of vector space structure prevents this and random perturbations are often most naturally expressed with sequences of infinitesimal steps. Vectors are thus replaced with tangent bundle valued sequences that, when integrated over time, give rise to stochastic flows. When modelling both deterministic and random variations, stochasticity generally couples non-trivially with the deterministic evolution. In addition, perturbations and correlation structure must be specified with respect to a frame of reference. While Euclidean space provides a global coordinate system allowing this, a model of transport must be specified in non-linear spaces. The stochastic metamorphosis model is an example of a model coupling deterministic and stochastic evolution and using right-invariance to provide reference frames for the perturbations and correlation structure. An example of a related but different approach is Kühnel and Sommer (2017) where parallel transport is used to link covariance between tangent spaces.

\section{Acknowledgements}

AA acknowledges funding from the EPSRC through award EP/N014529/1 funding the EPSRC Centre for Mathematics of Precision Healthcare. AA and DH are partially supported by the European Research Council Advanced Grant 267382 FCCA held by DH. DH is also grateful for partial support from EPSRC Grant EP/N023781/1. SS is partially supported by the CSGB Centre for Stochastic Geometry and Advanced Bioimaging funded by a grant from the Villum foundation. The authors would like to thank the Isaac Newton Institute for Mathematical Sciences for their support and hospitality during the programme Growth form and self-organisation when this paper was finished. This work is supported by EPSRC Grant Number EP/K032208/1.

\section{References}

Stephanie Allassonnière, Yali Amit, and Alain Trouvé. Towards a coherent statistical framework for dense deformable template estimation. Journal of the Royal Statistical Society: Series B (Statistical Methodology), 69(1):3-29, 2007. ISSN 1467-9868. doi: 10.1111/j.1467-9868.2007.00574.x. URL http://onlinelibrary.wiley.com/doi/10. 1111/j.1467-9868.2007.00574.x/abstract.

Alexis Arnaudon, Alex L Castro, and Darryl D Holm. Noise and dissipation on coadjoint orbits. To appear in JNLS, arXiv preprint arXiv:1601.02249, 2016.

Alexis Arnaudon, Darryl D Holm, and Stefan Sommer. A geometric framework for stochastic shape analysis. arXiv preprint arXiv:1703.09971, 2017.

M Faisal Beg, Michael I Miller, Alain Trouvé, and Laurent Younes. Computing large deformation metric mappings via geodesic flows of diffeomorphisms. International journal of computer vision, 61(2):139-157, 2005. 
Benjamin Charlier, Nicolas Charon, and Alain Trouvé. The Fshape Framework for the Variability Analysis of Functional Shapes. Foundations of Computational Mathematics, 17(2):287-357, 2017. ISSN 1615-3375, 1615-3383. doi: 10.1007/s10208-015-9288-2. URL https://link.springer.com/article/10.1007/s10208-015-9288-2.

Ming Chen, Youjin Zhang, et al. A two-component generalization of the camassa-holm equation and its solutions. Letters in Mathematical Physics, 75(1):1-15, 2006.

Gary E. Christensen, Richard Rabbitt, and Michael I. Miller. Deformable templates using large deformation kinematics. Image Processing, IEEE Transactions on, 5(10), 1996.

Paul Dupuis, Ulf Grenander, and Michael I. Miller. Variational Problems on Flows of Diffeomorphisms for Image Matching. Quarterly of applied mathematics, 1998.

P. Thomas Fletcher and Miaomiao Zhang. Probabilistic Geodesic Models for Regression and Dimensionality Reduction on Riemannian Manifolds. In Riemannian Computing in Computer Vision, pages 101-121. Springer, Cham, 2016. ISBN 978-3-319-22956-0 978-3-319-22957-7.

François Gay-Balmaz and Tudor S Ratiu. The geometric structure of complex fluids. Advances in Applied Mathematics, 42(2):176-275, 2009.

Darryl D Holm. Euler-Poincaré dynamics of perfect complex fluids. Geometry, mechanics, and dynamics, pages 169-180, 2002.

Darryl D Holm. Variational principles for stochastic fluid dynamics. Proceedings of the Royal Society of London A: Mathematical, Physical and Engineering Sciences, 471(2176): 20140963, 2015.

Darryl D Holm. Stochastic metamorphosis in imaging science. arXiv preprint arXiv:1705.10149, 2017.

Darryl D Holm and Tomasz M Tyranowski. Variational principles for stochastic soliton dynamics. In Proc. R. Soc. A, volume 472, page 20150827. The Royal Society, 2016.

Darryl D Holm, Alain Trouvé, and Laurent Younes. The Euler-Poincaré theory of metamorphosis. Quarterly of Applied Mathematics, 67(4):661-685, 2009.

Sarang Joshi, Brad Davis, Matthieu Jomier, and Guido Gerig. Unbiased diffeomorphic atlas construction for computational anatomy. NeuroImage, 23:151-160, 2004.

Line Kühnel and Stefan Sommer. Stochastic Development Regression on Non-linear Manifolds. In Information Processing in Medical Imaging, Lecture Notes in Computer Science, pages 53-64. Springer, Cham, June 2017. ISBN 978-3-319-59049-3 978-3-319-59050-9. doi: 10.1007/978-3-319-59050-9_5.

Line Kühnel, Stefan Sommer, Aksshha Pai, and Lars Raket. Most Likely Separation of Intensity and Warping Effects in Image Registration. SIAM Journal on Imaging Sciences, 10(2):578-601, 2017. doi: 10.1137/16M1070980.

J. S. Marron, James O. Ramsay, Laura M. Sangalli, and Anuj Srivastava. Functional Data Analysis of Amplitude and Phase Variation. Statistical Science, 30(4):468-484, 2015. ISSN 0883-4237, 2168-8745. doi: 10.1214/15-STS524. 
Stephen Marsland and Tony Shardlow. Langevin equations for landmark image registration with uncertainty. SIAM Journal on Imaging Sciences, 10(2):782-807, 2017.

Michael I. Miller and Laurent Younes. Group actions, homeomorphisms, and matching: A general framework. International Journal of Computer Vision, 41(1-2):61-84, 2001.

Prasanna Muralidharan and P. Thomas Fletcher. Sasaki Metrics for Analysis of Longitudinal Data on Manifolds. Proceedings / CVPR, IEEE Computer Society Conference on Computer Vision and Pattern Recognition. IEEE Computer Society Conference on Computer Vision and Pattern Recognition, 2012:1027-1034, 2012. ISSN 1063-6919. doi: 10.1109/CVPR.2012.6247780.

Marc Niethammer, Yang Huang, and FranÃğois-Xavier Vialard. Geodesic Regression for Image Time-Series. In Medical Image Computing and Computer-Assisted Intervention $\hat{a} \breve{A} S$ MICCAI 2011, Lecture Notes in Computer Science, pages 655662. Springer, Berlin, Heidelberg, 2011. ISBN 978-3-642-23628-0 978-3-642-23629-7. doi: 10.1007/978-3-642-23629-7_80. URL https://link.springer.com/chapter/10. 1007/978-3-642-23629-7_80.

Akshay Pai, Stefan Sommer, Lars Lau Raket, Line Kühnel, Sune Darkner, Lauge SÃÿrensen, and Mads Nielsen. A Statistical Model for Simultaneous Template Estimation, Bias Correction, and Registration of 3d Brain Images. 2016.

Lars Lau Raket, Stefan Sommer, and Bo Markussen. A nonlinear mixed-effects model for simultaneous smoothing and registration of functional data. Pattern Recognition Letters, $38: 1-7,2014$.

Stefan Sommer. Anisotropic Distributions on Manifolds: Template Estimation and Most Probable Paths. In Information Processing in Medical Imaging, volume 9123 of Lecture Notes in Computer Science, pages 193-204. Springer Berlin Heidelberg, 2015.

Stefan Sommer and Anne Marie Svane. Modelling anisotropic covariance using stochastic development and sub-Riemannian frame bundle geometry. Journal of Geometric Mechanics, 9(3):391-410, 2017. ISSN 1941-4889. doi: 10.3934/jgm.2017015.

Alain Trouvé. An infinite dimensional group approach for physics based models in pattern recognition. preprint, 1995.

Alain Trouvé and François-Xavier Vialard. Shape splines and stochastic shape evolutions: a second order point of view. Quarterly of Applied Mathematics, 70(2):219-251, 2012.

Alain Trouvé and Laurent Younes. Local geometry of deformable templates. SIAM journal on mathematical analysis, 37(1):17-59, 2005a.

Alain Trouvé and Laurent Younes. Metamorphoses through lie group action. Foundations of Computational Mathematics, 5(2):173-198, 2005b.

J. Derek Tucker, Wei Wu, and Anuj Srivastava. Generative models for functional data using phase and amplitude separation. Computational Statistics \& Data Analysis, 61 (Supplement C):50-66, 2013. ISSN 0167-9473. doi: 10.1016/j.csda.2012.12.001. URL http://www.sciencedirect.com/science/article/pii/S0167947312004227. 
François-Xavier Vialard. Extension to infinite dimensions of a stochastic second-order model associated with shape splines. Stochastic Processes and their Applications, 123 (6):2110-2157, 2013.

Laurent Younes, Felipe Arrate, and Michael I. Miller. Evolutions equations in computational anatomy. NeuroImage, 45(1, Supplement 1):S40-S50, 2009. ISSN 1053-8119. doi: 10.1016/j.neuroimage.2008.10.050.

Miaomiao Zhang, Nikhil Singh, and P Thomas Fletcher. Bayesian estimation of regularization and atlas building in diffeomorphic image registration. In International Conference on Information Processing in Medical Imaging, pages 37-48. Springer, 2013. 